EXTENDED REPORT

\title{
Analysis of p63 and cytokeratin expression in a cultivated limbal autograft used in the treatment of limbal stem cell deficiency
}

\author{
D G Harkin, Z Barnard, P Gillies, S L Ainscough, A J G Apel
}

See end of article for authors' affiliations

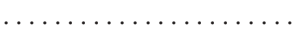

Correspondence to: Dr D G Harkin, Tissue BioRegeneration and Integration Program, Institute for Health and Biomedical Innovation, Queensland University of Technology, 2 George Street, Brisbane 4000, Australia; d.harkin@qut.edu.au

Accepted 1 January 2004
Br J Ophthalmol 2004;88:1154-1158. doi: 10.1136/bjo.2003.037853

Aim: To investigate the expression of p63 and cytokeratins throughout the course of producing a cultivated autograft of limbal epithelial cells.

Methods: A 75 year old male with a severe alkali burn to his right eye received two cultivated autografts of limbal epithelial cells on amniotic membrane followed by a corneal allograft. Immunostaining for p63 and cytokeratins was performed during ex vivo expansion with 3T3 fibroblasts, following subcultivation on amniotic membrane, and on the excised corneal button.

Results: Cultures grown in the presence of 3T3 fibroblasts or on amniotic membrane displayed positive staining for keratins 14 and 19, and p63, but poor staining for keratin 3 (K3). The excised corneal button possessed a stratified epithelium of K3 positive cells residing on amniotic membrane.

Conclusions: Our results document for the first time the co-expression of cytokeratins 14 and 19 with p63 in a cultivated limbal graft. These data support the conclusion that cultivated grafts of limbal epithelium contain predominantly undifferentiated cells with the potential to regenerate a normal corneal epithelium.

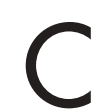
ultivated grafts of limbal epithelial cells are emerging as a valuable adjunct therapy for repairing the ocular surface. ${ }^{1-7}$ The rationale behind this treatment is that the limbal epithelium contains adult stem cells or progenitor cells for regenerating the corneal epithelium. Nevertheless, this technique is still experimental and as such there is little consensus as to the best way to grow, graft, and validate the phenotype of limbal cultures. Thus in the course of treating a patient with two cultivated autografts of limbal epithelial cells we took the opportunity to examine the phenotype of the patient's cells at three critical stages: (1) during ex vivo expansion in the presence of growth arrested mouse 3 T3 fibroblasts; (2) following subcultivation on a cryopreserved carrier substrate of donor amniotic membrane, and (3) following application to the ocular surface.

It is generally assumed that the efficacy of grafted cultures is due to the presence of corneal stem cells, but in the absence of a definitive marker their role remains unclear. Nevertheless, a number of proteins, for which antibodies are readily available, provide valuable markers for assessing limbal epithelial cell phenotype. For example, keratin 3 (K3) is expressed by limbal epithelial cells as they begin to differentiate and is retained in the corneal epithelium. ${ }^{8}$ In addition, keratins 14 and 19 are produced by basal limbal epithelial cells and thus are considered to be associated with the progenitor cell phenotype. ${ }^{9-11}$ More recently, the transcription factor p63 has been identified as a putative keratinocyte stem cell marker for both the epidermis and corneal epithelium. ${ }^{12}$ However, earlier analyses of cultivated limbal grafts used in clinical studies have been limited primarily to studies of $\mathrm{K} 3$ expression. ${ }^{1457}$ Our study is therefore the first to document the expression of p63 in conjunction with keratins 3, 14, and 19, during production of a cultivated limbal graft.

\section{MATERIALS AND METHODS}

A 75 year old male presented with a 10 year old injury to his right eye caused by a severe alkali burn (fig 1A). Over $50 \%$ of the ocular surface was covered by conjunctival tissue with extensive scarring and vascularisation extending into the underlying stroma. Based on the severity of stem cell failure in the injured eye (stage IIb), ${ }^{13}$ and the presence of a healthy non-injured left eye, the patient was judged to be a suitable candidate for a cultivated limbal autograft. The experimental nature of this procedure was explained to the patient, and signed consent obtained beforehand. Ethical approval was also obtained from the human research ethics committees of all associated institutions.

Cultivated grafts of limbal epithelial cells have been produced either from tissue explants grown directly on amniotic membrane $e^{367}$ or from dissociated cultures expanded ex vivo in the presence of growth arrested 3T3 fibroblasts. $^{125}$ In the present study, we used ex vivo expansion as it provides sufficient cells for multiple grafts and thus negates the requirement for further biopsies. Although the precise role of $3 \mathrm{~T} 3$ fibroblasts is unclear, the survival and proliferation of epithelial progenitor cells is supported by interactions with the mouse derived cells and/or their secreted products. ${ }^{14}$

Two biopsies of approximately $2 \mathrm{~mm}^{2}$ each were removed from the patient's healthy left eye in theatre under local anaesthetic and immediately transported to the culture facility in sterile phosphate buffered saline (PBS). The biopsies were obtained from the superior and inferior limbal margins respectively (identified by limbal palisades) and extended approximately $0.5 \mathrm{~mm}$ into the peripheral cornea and adjacent conjunctiva. All subsequent handling of patient tissue and cells was carried out within a Class II biohazard hood. The two samples were combined and dissociated by gentle trituration following incubation for 10 minutes at $37^{\circ} \mathrm{C}$ in PBS containing $0.25 \%$ trypsin and $1 \mathrm{mM}$ ethylene diamine tetra-acetic acid (EDTA). Residual trypsin activity was inactivated by washing and resuspending in culture medium

Abbreviations: EDTA, ethylene diamine tetra-acetic acid; FBS, fetal bovine serum; K3, keratin 3; PBS, phosphate buffered saline. 

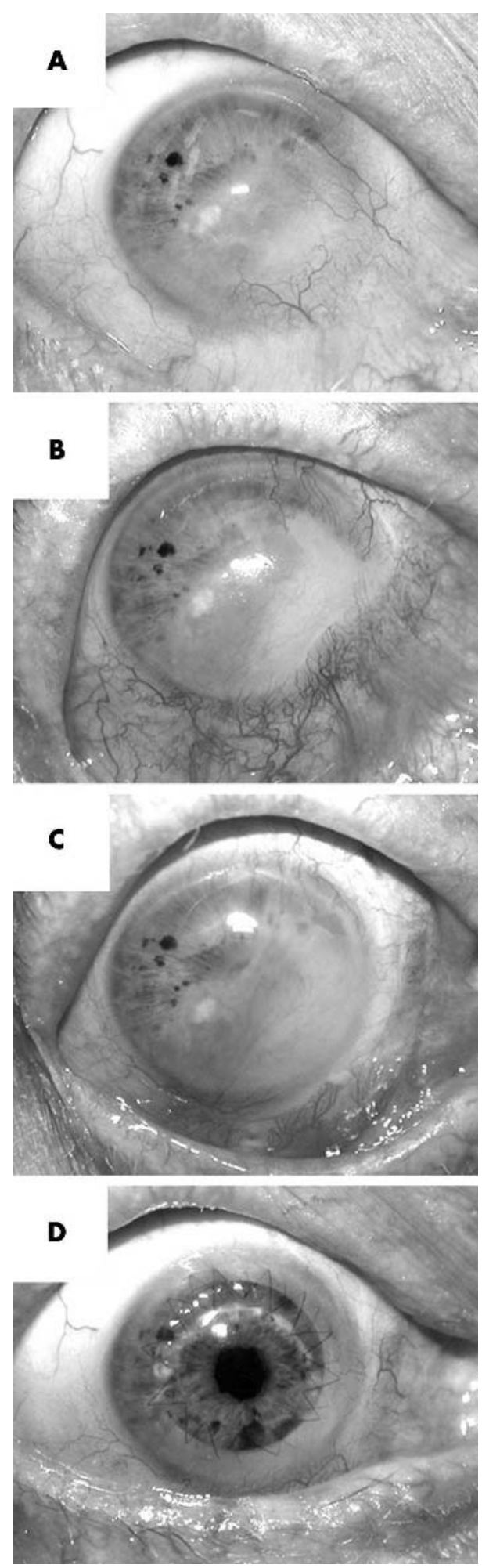

Figure 1 Condition of patient's injured eye before and after treatments. (A) Appearance on presentation 10 years after injury; (B) 4 months after application of the first cultivated graft; (C) 2 months after application of the second cultivated graft, and (D) 3 months after penetrating keratoplasty. The time between applying the initial cultivated graft and the final photograph is approximately 15 months.

(DMEM/F12 medium, $1 \mu \mathrm{g} / \mathrm{ml}$ insulin, $0.4 \mu \mathrm{g} / \mathrm{ml}$ hydrocortisone, $10 \mathrm{ng} / \mathrm{ml}$ epidermal growth factor, $180 \mu \mathrm{M}$ adenine, $10 \mathrm{ng} / \mathrm{ml}$ cholera toxin) supplemented with $10 \%$ gamma irradiated fetal bovine serum (FBS) from Australian/New
Zealand herds (Trace, Melbourne, Australia). Recovered cells were passaged twice in the presence of gamma irradiated mouse 3 T3 fibroblasts $\left(5 \times 10^{4} / \mathrm{cm}^{2}\right)$ over a period of 2 weeks resulting in approximately $10^{7}$ cells. The initial culture (P0) was established in a $35 \mathrm{~mm}$ diameter culture dish and the secondary culture (Pl) was grown in a $75 \mathrm{~cm}^{2}$ flask. A sample of these cells was subcultivated $\left(2 \times 10^{4} / \mathrm{cm}^{2}\right)$ in a 24 well culture plate containing $13 \mathrm{~mm}$ diameter glass cover slips for a further 3 days in the presence of 3T3 fibroblasts $\left(5 \times 10^{4} / \mathrm{cm}^{2}\right)$ and processed for immunostaining (fig 2). Two additional tertiary cultures (P2) were established at this time on cryopreserved amniotic membrane at a density of $2 \times 10^{5}$ cells $/ \mathrm{cm}^{2}$ and cultured for 2 weeks in the presence of $10 \%$ FBS. The amniotic epithelium was removed before seeding limbal cells using $0.25 \%$ trypsin/ $/ \mathrm{mM}$ EDTA $\left(37^{\circ} \mathrm{C}\right.$ for 10 minutes) and a plastic cell scraper. One P2 culture on amniotic membrane was processed for immunohistochemistry (fig 3), and the other grafted onto the patient's injured eye as follows. Surface scar tissue was removed by scraping and the conjunctival epithelium recessed 3-4 mm back beyond the limbal boundary. The cultivated graft was then applied face up across affected regions of ocular surface from limbus to limbus, with sutures placed into the abutting recessed conjunctiva and underlying sclera, and covered for 2 months with a contact lens. The outcome of this initial grafting is shown in figure 1B. A second graft of cells on amniotic membrane was prepared and applied approximately 5 months later to two quadrants with residual conjunctival overgrowth (outcome shown in fig $1 \mathrm{C}$ ). The second graft was prepared from the patient's own excess passaged cells (P2) that had been cryopreserved in $90 \% \mathrm{FBS} / 10 \%$ dimethylsulfoxide (DMSO). These cells were thawed and passaged once in the presence of 3T3 cells to ensure viability before seeding onto amniotic membrane (that is, P3 cells were grafted). Penetrating keratoplasty was performed 7 months after the second cultivated graft to treat stromal scarring (outcome shown in fig 1D) and the excised corneal button retained for immunostaining. Immunostaining of limbal cultures and sectioned material was performed as described previously. ${ }^{11}$ Antibodies to keratins 3 (AE5), 14 (LL002), and 19 (BA17) were obtained from Research Diagnostics (Flanders, NJ, USA). The 4A4 monoclonal antibody to p63 was purchased from Labvision Neomarkers (Edward Keller Pty Ltd, Brisbane, Australia). An Alexa-fluor 488 goat-antimouse secondary antibody was used (Molecular Probes, Eugene, OR, USA). Each marker was examined separately in replicate cultures and serial sections.

\section{RESULTS}

During the first few days of coculture, islands of small and tightly packed limbal epithelial cells emerge amid the near confluent layer of $3 \mathrm{~T} 3$ fibroblasts. In time, the limbal cell islands begin to merge and a degree of stratification is observed towards the centre. After approximately one week, the majority of 3T3 fibroblasts are displaced from the culture dish and the patient cells are ready for passaging in the presence of freshly irradiated 3T3 fibroblasts. Approximately 10 million patient cells are produced by the time the secondary culture reaches confluency. We have examined the phenotype of these cultures after reseeding for 3 days in the continued presence of freshly irradiated $3 \mathrm{~T} 3$ fibroblasts. The resulting pattern of growth is similar to that seen in primary and secondary cultures with small islands of limbal cells once more being apparent (fig 2A). Immunocytochemistry indicates that the majority of cells in these tertiary cultures $(>95 \%)$ express the putative keratinocyte stem cell marker p63 (fig 2C), as well as keratins 14 (fig 2D) and 19 (fig 2E). Some immunoreactivity towards the AE5 antibody to K3 is also observed (approximately $85 \%$ of 

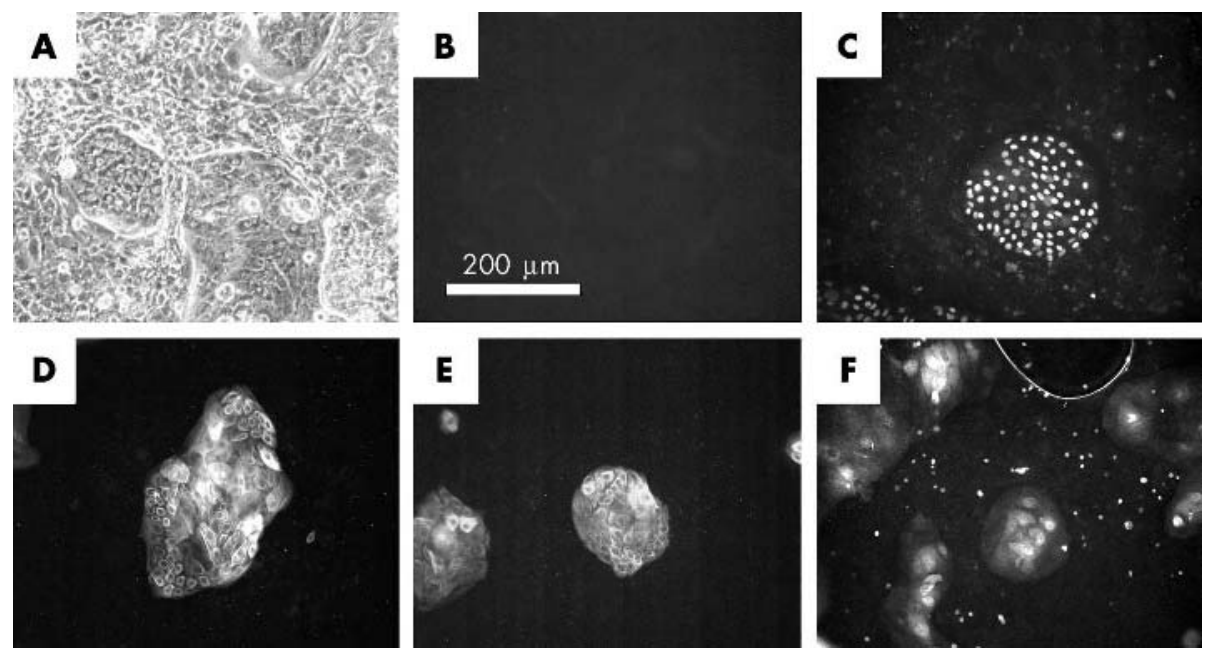

Figure 2 Phenotypic analysis of limbal epithelial cells co-cultured with mouse 3T3 cells. A sample of excess patient cells from the secondary culture was immunostained for p63 and cytokeratins after culturing for an additional 3 days in the presence of freshly irradiated mouse 3T3 cells. (A) Phase contrast image displaying compact morphology of limbal islands amid 3T3 cells. (B) Negative control for all primary antibodies used, incubated with secondary antibody alone. (C) Island stained with 4A4 monoclonal antibody to p63. (D) Island stained with LLO02 monoclonal antibody to keratin 14. (E) Islands stained with BA17 monoclonal antibody to keratin 19. (F) Islands stained with AE5 antibody to keratin 3. Each photographed island is representative of the majority of islands present in each immunostained sample.

islands), but is restricted to stratifying cells near the centre of each island (fig 2F). A similar profile of staining is observed for cells derived from the same secondary culture when subcultured for 2 weeks on donor amniotic membrane. In brief, an epithelium of one to two layers is observed with most cells reacting with antibodies to p63, keratin 14, and keratin 19. In contrast, reactivity towards the AE5 antibody to $\mathrm{K} 3$ is poor (data not shown). Two similar cultures of cells on amniotic membrane were grafted. The first was grown in duplicate to the culture examined by immunostaining, and the second was prepared 5 months later using excess cells from the patient's secondary culture that had been stored in liquid nitrogen. The fate of these grafted cultures was investigated following subsequent penetrating keratoplasty. Sections were obtained from across the widest part of the excised corneal button to ensure sampling of tissue originally covered by conjunctiva. Two observations were made during this analysis. Firstly, the surface of the excised button is covered by a stratified epithelium of approximately six layers that stains positively for keratin 3 (fig $3 \mathrm{H}$ ), but not keratin 14, keratin 19, or p63 (not shown). Reactivity to the keratin 3 antibody AE5 is greatest in the superficial layers and extends across the width of the excised corneal button. Secondly, subepithelial material is observed that resembles the thick basement membrane of the amniotic membrane.

\section{DISCUSSION}

An increasing number of papers have shown the effectiveness of cultivated limbal grafts for reconstructing the cornealconjunctival boundary. ${ }^{1-7}$ This opinion is further supported by the present study in which two cultivated grafts were applied in an effort to stabilise the ocular surface prior to penetrating keratoplasty (fig 1). The future for cultivated limbal grafts as an adjunct therapy to corneal allografts therefore appears promising. Nevertheless, advancement of the technology beyond its present experimental status will require cultivated
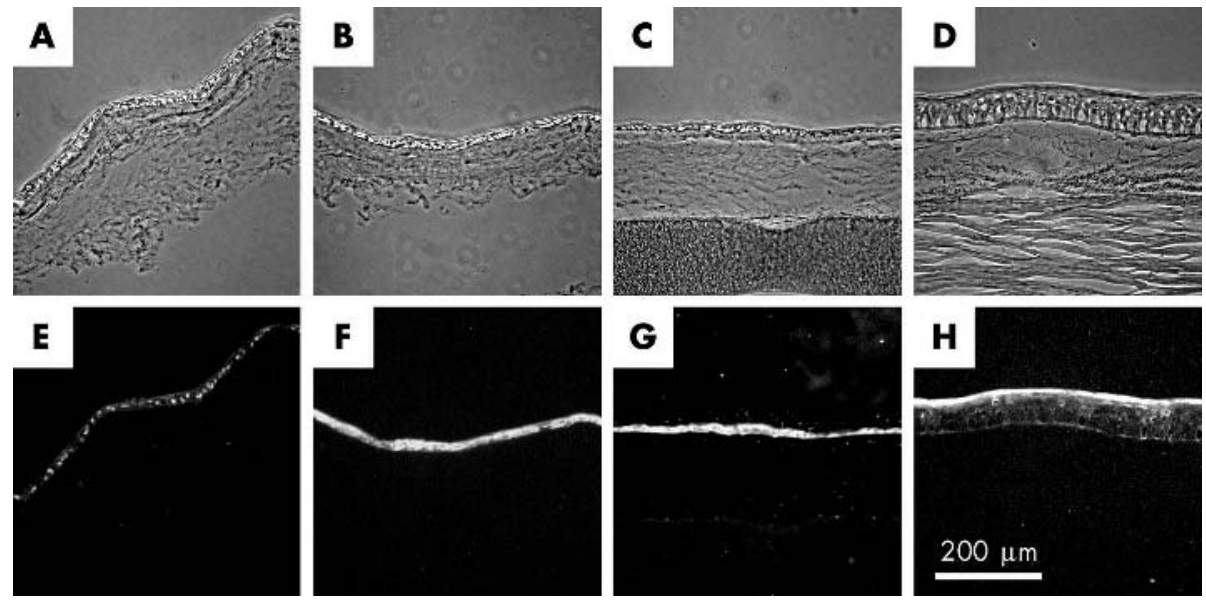

Figure 3 Phenotypic analysis of cultures grown on amniotic membrane, and corneal button recovered during subsequent keratoplasty. Patient cells derived from the same culture as those used in figure 2 were cultivated for 2 weeks on two pieces of denuded amniotic membrane. (A-C) Sections obtained from the non-grafted duplicate culture. A piece of backing paper remains attached to the section in part C. (D) A section obtained from the patient's excised corneal button obtained following keratoplasty. Note the presence of material resembling the amniotic basement membrane between the corneal stroma and regenerated epithelium. (E-G) The same sections of cultivated graft as in parts A-C but stained for p63, keratin 14, and keratin 19 respectively. $(H)$ The same section of excised corneal button as in part D stained for keratin 3. 
limbal grafts to comply with regulatory guidelines for cell based treatments that are currently under review in many countries including Australia. In essence, the emerging legislation is aimed at ensuring adequate control over the manufacturing process and validation of the product before release for clinical use. To this end, during the present study we chose to investigate the expression of a novel combination of markers that might be used to validate the phenotype of cultivated limbal grafts-namely cytokeratins 3, 14, and 19, and p63. Moreover, we followed the expression of each protein at critical stages during culture and following engraftment to the ocular surface.

Keratin 3 (K3) is absent from the basal layers of the limbal epithelium, but expressed in the mature corneal epithelium. ${ }^{8}$ Earlier analyses of cultivated limbal grafts have therefore used the AE5 antibody to $\mathrm{K} 3$ in order to validate growth of the correct cell type in vitro and following grafting. ${ }^{1457}$ Nevertheless, the discovery of $\mathrm{K} 3$ in a patient's cultivated graft presents a dilemma as high levels of keratin 3 expression theoretically indicate that cultures are more differentiated and therefore less likely to retain proliferative properties once applied to the ocular surface. The low levels of K3 observed in the present study are therefore consistent with a poorly differentiated population of progenitor cells for the corneal epithelium. Furthermore, the widespread expression of $\mathrm{K} 3$ in the corneal button retrieved following keratoplasty is useful for confirming recovery of a normal ocular surface. The presence of material resembling amniotic membrane beneath the reconstituted epithelium suggests that grafted cells are responsible for the recovery, but it remains possible that existing cells may have emigrated from the superior temporal quadrant which was less injured. In either case, the grafted cells may create an environment that encourages existing progenitor cells to aid repair of the ocular surface.

Keratins 14 and 19 (K14 and K19) are found in many epithelial tissues including skin and conjunctiva and thus have limited potential as markers for limbal cells and their progeny. Nevertheless, K14 is associated with hemidesmosome formation and thus has been used to study the attachment of limbal cell cultures to sheets of amniotic membrane. ${ }^{15}$ The expression of K14 observed in monolayers of limbal cells either grown in the presence of 3T3 cells or on amniotic membrane is therefore consistent with the normal behaviour of basal epithelial cells. The parallel expression of $\mathrm{K} 19$ is likewise significant as this protein is more closely associated with the location of progenitor cells in both skin and the limbus. ${ }^{10} 16$ The co-expression of K14 and K19 is therefore useful as a means for identifying epithelial progenitor cells with basal cell activities. The absence of K14 and K19 in the excised corneal button can be considered in several ways. Firstly, the result is consistent with the hypothesis that the grafted cells differentiated following application to the ocular surface. Secondly, the absence of K19 confirms that conjunctival cells have not re-emigrated across the ocular surface. ${ }^{5}$ Keratin 19 expression in the original 3T3 co-cultures is unlikely to be due to contaminating conjunctival cells as these grow poorly in culture, and the majority of islands contain $\mathrm{K} 3$ positive cells at their centre.

Given the limitations of keratins as markers for limbal stem cells, other molecules have been investigated as potential markers including the nuclear transcription factor, p63. p63 is an intriguing molecule because it belongs to the same family of proteins as the tumour suppressor gene, $\mathrm{p} 53 .{ }^{17}$ Nevertheless, unlike its relative, p63 is associated with the development of tissues, especially stratified epithelia and their functional derivatives. ${ }^{18}$ Based on these observations, Pellegrini et al hypothesised that p63 is required to support the phenotype of epithelial stem cells in both skin and the cornea. ${ }^{12}$ Their subsequent studies of the ocular surface revealed a localised expression of p63 within the basal epithelial cells of the limbus. Moreover, a detailed clonal analysis demonstrated a strong correlation between p63 expression and the proliferative potential of limbal epithelial cells in vitro. The present study extends this knowledge by demonstrating the co-expression of keratins 14 and 19 with p63 in vitro. This co-expression is inferred by the high percentage of stained cells observed in replicate cultures. Moreover, we have performed this analysis on cultures that have been applied to the ocular surface. In considering the significance of our data it should be noted that although p63 is associated with the phenotype of holoclones (stem cells), lesser amounts have been detected in their immediate progeny (meroclones, young transient amplifying cells) in vitro $^{12}$ and within the peripheral cornea in situ. ${ }^{19}$ It is therefore likely, given the widespread staining observed in our study, that the majority of p63 positive cells are primitive cells resulting from the division and differentiation of limbal stem cells. Nevertheless, it should also be noted that the 4A4 monoclonal antibody used to detect p63 expression recognises several isoforms in addition to the truncated $\Delta \mathrm{Np} 63 \alpha$ form that is reportedly associated with epithelial stem cells. The full potential of p63 as a marker for limbal epithelial cell lineage has therefore yet to be established. In the meantime, p63 remains a useful molecule for validating the phenotype of cultivated limbal grafts given that it is associated with a proliferative, poorly differentiated phenotype.

In summary, our study documents for the first time the coexpression of cytokeratins and p63 in the course of producing a cultivated autograft of limbal epithelial cells. The results of this analysis are consistent with the hypothesis that cultures grown in the presence of $3 \mathrm{~T} 3$ cells or on amniotic membrane contain predominantly poorly differentiated progenitor cells for the corneal epithelium. Nevertheless, consideration must be given to the limited specificity of markers for cytokeratins and p63 when validating the phenotype of limbal cultures.

\section{ACKNOWLEDGEMENTS}

We are grateful for financial assistance from the following: Royal Brisbane and Women's Hospital Foundation, Ophthalmic Research Institute of Australia, OPSM Charitable Research Fund, and $\mathrm{Mr}$ Maxwell Perrins, Clayfield, Queensland. Additional resources were supplied by the Queensland Eye Centre, Spring Hill, Brisbane. We also acknowledge the Lions Corneal Donation Service, Royal Victoria Eye \& Ear Hospital, Melbourne for supplying donor amniotic membrane.

\section{Authors' affiliations}

D G Harkin, P Gillies, S L Ainscough, Tissue BioRegeneration and Integration Program, Institute for Health and Biomedical Innovation, Queensland University of Technology, Brisbane, Queensland, Australia Z Barnard, School of Biomolecular \& Biomedical Science, Griffith University, Brisbane, Queensland, Australia

P Gillies, Queensland Skin Bank, Australian Red Cross Blood Service, Brisbane, Queensland, Australia

A J G Apel, Princess Alexandra Hospital, Brisbane, Queensland, Australia

\section{REFERENCES}

1 Pellegrini G, Traverso C, Franzi A, et al. Long-term restoration of damaged corneal surfaces with autologous cultivated corneal epithelium. Lancet 1997;349:990-3.

2 Schwab I. Cultured corneal epithelia for ocular surface disease. Trans Am Ophthalmol Soc 1999;97:891-986.

3 Tsai R-F, Li L-M, Chen J-K. Reconstruction of damaged corneas by transplantation of autologous limbal epithelial cells. N Engl J Med 2000;343:86-93.

4 Koizumi N, Inatomi T, Suzuki T, et al. Cultivated corneal epithelial stem cell transplantation in ocular surface disorders. Ophthalmology 2001;108:1569-74. 
5 Rama P, Bonini S, Lambiase A, et al. Autologous fibrin-cultured limbal stem cells permanently restore the corneal surface of patients with total limbal stem cell deficiency. Transplantation 2001;72:1478-85.

6 Shimazaki J, Aiba M, Goto E, et al. Transplantation of human limbal epithelium cultivated on amniotic membrane for the treatment of severe ocular surface disorders. Ophthalmology 2002;109:1285-90.

7 Grueterich M, Espana EM, Touhami A, et al. Phenotypic study of a case with successful transplantation of ex vivo expanded human limbal epithelium for unilateral total limbal stem cell deficiency. Ophthalmology

2002; 109:1547-52.

8 Schermer A, Galvin S, Sun T-T. Differentiation-related expression of a major $64 \mathrm{~K}$ corneal keratin in vivo and in culture suggests limbal location of corneal epithelial stem cells. J Cell Biol 1986;103:49-62.

9 Kasper M, Moll R, Stosiek P, et al. Patterns of cytokeratin and vimentin expression in the human eye. Histochemistry 1988;89:369-73.

10 Lauweryns B, van den Oord J, De Vos R, et al. A new epithelial cell type in the human cornea. Invest Ophthalmol Vis Sci 1993;34:1983-90.

11 Barnard Z, Apel J, Harkin D. Phenotypic analyses of limbal epithelial cell cultures derived from donor corneal scleral rims. Clin Experiment Ophthalmol 2001:29:138-42.

12 Pellegrini G, Dellambra E, Golisano O, et al. p63 identifies keratinocyte stem cells. Proc Natl Acad Sci U S A 2001;98:3156-61.
13 Holland E Schwartz G. The evolution of epithelial transplantation for severe ocular surface disease and a proposed classification system. Cornea 1996; 15:549-69.

14 Rheinwald J, Green H. Serial cultivation of strains of human epidermal keratinocytes: the formation of keratinizing colonies from single cells. Cell 1975:6:331-43.

15 Meller D, Pires R, Tseng S. Ex vivo preservation and expansion of human limbal epithelial stem cells on amniotic membrane cultures. Br J Ophthalmol 2002:86:463-71.

16 Michel M, Torok N, Godbout M, et al. Keratin 19 as a biochemical marker of skin stem cells in vivo and in vitro: keratin 19 expressing cells are differentially localized in function of anatomic sites, and their number varies with donor age and culture stage. J Cell Sci 1996; 109:1017-28.

17 Little N, Jochemsen A p63. Int I Biochem Cell Biol 2002:34:6-9.

18 Yang A, Schweitzer R, Sun D, et al. p63 is essential for regenerative proliferation in limb, craniofacial and epithelial development. Nature 1999:398:714-18.

19 Hernandez Galindo E, Theiss C Steuhl K-P et al. Expression of $\Delta \mathrm{Np} 63$ in response to phorbol ester in human limbal epithelial cells expanded on intact human amniotic membrane. Invest Ophthalmol Visual Sci 2003;44:2959-65 